\title{
Characterization of Mo/Si multilayer growth on stepped topographies
}

A. J. R. van den Boogaard, ${ }^{\text {a) }}$ E. Louis, and E. Zoethout

FOM Institute for Plasma Physics Rijnhuizen, P.O. Box 1207, NL-3430 BE, Nieuwegein, The Netherlands

K. A. Goldberg

Center for X-Ray Optics, Lawrence Berkeley National Laboratory, Berkeley, California 94720, USA

F. Bijkerk

FOM Institute for Plasma Physics Rijnhuizen, P.O. Box 1207,3430 BE, Nieuwegein, The Netherlands and MESA+ Institute for Nanotechnology, University of Twente, P.O. Box 217, NL-7500AE, Enschede

$\mathrm{Mo} / \mathrm{Si}$ multilayer mirrors with nanoscale bilayer thicknesses have been deposited on stepped substrate topographies, using various deposition angles. The multilayer morphology at the stepedge region was studied by cross section transmission electron microscopy. A transition from a continuous- to columnar layer morphology is observed near the step-edge, as a function of the local angle of incidence of the deposition flux. Taking into account the corresponding kinetics and anisotropy in layer growth, a continuum model has been developed to give a detailed description of the height profiles of the individual continuous layers. Complementary optical characterization of the multilayer system using a microscope operating in the extreme ultraviolet wavelength range, revealed that the influence of the step-edge on the planar multilayer structure is restricted to a region within $300 \mathrm{~nm}$ from the step-edge. ${ }^{\mathrm{VC}} 2011$ American Vacuum Society.

\section{INTRODUCTION}

The optical characteristics of nanoscale thin film multi- layer structures deposited on substrates with a strictly controlled surface figure and roughness are subject to numerous studies. For semiflat superpolished substrates (rms roughness $\leq 0.1 \mathrm{~nm}$ ) the alternating parallel layers may serve as a Bragg-reflector for extreme ultraviolet (EUV) or soft $\mathrm{x}$ ray radiation, yielding high specular reflectance in near-normal conditions. Multilayer structures deposited on 3D patterned substrate topographies have great potential as high efficiency diffractive optics, e.g., applicable as a monochromator ${ }^{1}$ or in high resolution spectrometry, ${ }^{2,3}$ and

as reflective phase-mask for EUV lithography. ${ }^{4}$ Recent studies mainly focus on fabrication methods of suited substrate topographies such as gratings which meet the Bragg-reflector imposed quality demands on roughness levels. ${ }^{1,2,5}$ However, the optical response of mentioned systems depends on the replication efficiency of a predefined substrate topography in the deposited multilayer structure. Near key features like step-edges the multilayers will show deformations from the desired, i.e., locally planar, structure. This causes spectral and spatial shifts in the optical response and general reflectance losses, which will be of increasing significance at downscaled pitch dimensions. Detailed knowledge on near stepedge layer growth is therefore required to gain understanding of the performance of diffractive optical multilayer devices, and indicate routes to further optimization. Besides, the findings might be of relevance to other fields of research concerned with the morphology of deposited nanostructures as physically synthesized multilayer nanoparticles. ${ }^{6}$

Substrate replication in thin films deposited by physical layer deposition at room temperature show generic dependence on two deposition parameters: geometrical factors and the energy of the adparticles. ${ }^{7,8}$ Hereby, the substrate replication direction and the substrate profile relaxation are determined, respectively. For the purpose of separately addressing these dependencies, it is desirable to use a well- controlled and isotropic deposition flux and a surface relaxation treatment decoupled from the primary layer growth process. In this paper Mo/Si multilayers, optimized on EUV reflectance, are deposited under various angles near substrate step-edges via electron-beam physical vapor deposition (e-beam PVD) and subsequent low energy $\mathrm{Kr}^{+}$-ion exposure. The individual bilayer profiles in the step-edge region are studied by cross section transmission electron microscopy (cs-TEM). To characterize the samples with high sensitivity for the multilayer periodicity, and over a lateral area extending the range of cs-TEM, the Actinic Inspection Tool (AIT) (Ref. 9) at the Berkeley Centre for X-ray Optics is used. This state of the art EUV microscopes provides a sub- $100 \mathrm{~nm}$ spatial resolution in the $\lambda=$ $13.5 \mathrm{~nm}$ wavelength range. 


\section{LAYER GROWTH EVOLUTION EQUATIONS}

The data are discussed in terms of a model description of the bilayer profiles throughout the multilayer structure. The continuum formalism as proposed by Stearns et al. is used. 10 The layer height profiles are given by $h_{N}(x)$ at bilayer number $\mathrm{N}$, as measured from the plane of the substrate with surface normal $\vec{z}$, and $\vec{z} \perp \vec{x}$. Only normal incidence deposition is considered in reference, ${ }^{10}$ with the surface normal of the layer growth front $\vec{n}$ is parallel to $\vec{z}$. The layer profile evolution is given by the recursion relation:

$h_{N+1}(f)=F\left[h_{N}(x)+d+\partial_{n l}(x)\right] \exp \left[-t_{\text {sput }} \times b(f)\right]$,

$h_{N+1}(x)=F^{*}\left[h_{N+1}(f)\right]$,

Where $F[\ldots]$ and $F^{*}[\ldots]$ indicate a Fourier transform and its inverse, respectively, and

$b(f)=\sum_{i} v_{i}(2 \pi f)^{i}$

Equation (1) gives the combined effect of layer growth by one-to-one replication with offset value $d$ indicating the bilayer thickness i.e. $d$-spacing, and $\partial_{n l}(x)$ nonlinear terms depending on local surface derivatives. The exponent can be considered as a damping term on the Fourier components defining the layer profile at the spatial frequency $(f)$ domain, for proportionality coefficients $v_{i}$ as associated with ion enhanced layer growth kinetics during sputtering over a thickness $t_{\text {sput }}$. In order to address the direction of the linear and nonlinear layer growth as a variable, the authors here propose the following basic substitution:

$$
d+\partial_{n l}(x) \rightarrow \vec{z} \cdot \mathbf{l} \vec{n}+\vec{\partial}_{n l}(x)
$$

And

$$
\widetilde{C}_{\Delta+1}=\vec{t}+\vec{x} \cdot\left(d \vec{n}+\vec{\partial}_{n l}(x)\right)_{\underline{N}}
$$

An explanatory model of the experimental data is based on the above general equations. The development and further implications imposed by the experimental conditions under consideration are described in Sec. III C.

\section{EXPERIMENTAL}

\section{A. Multilayer deposition}

Commercially available polished crystalline $\mathrm{Si}(100)$ wafers were cleaved parallel to a wafer flat, to serve as substrates for high reflective EUV Mo/Si multilayers. The cleaving process produces well defined, rectangular sample edges near which the multilayer morphology is studied. Mo/Si multilayers with 50 bilayers of $6.95 \mathrm{~nm}$ thickness and a Mo to (Mo+Si) thickness ration of 0.4 were deposited. Physical vapor deposition at room temperature and high-vacuum conditions with a base pressure of $10^{-9} \mathrm{mbar}$ have been employed. Surface smoothening was obtained by a $130 \mathrm{eV} \mathrm{Kr}$ ion polishing treatment after deposition of each Si layer, during which an excess layer of $0.5 \mathrm{~nm}$ Si was removed by physical sputtering. The Kaufmann ion-source was mounted under an 
angle of $45^{\circ}$ with respect to the axis of substrate rotation. The described deposition conditions and multilayer composition yield amorphous Si and nanocrystaline Mo layers. ${ }^{11}$

The deposition geometry was controlled by using a electron beam generated spatially confined melt of target material with a typical diameter of $2 \mathrm{~cm}$ to produce the evaporation plume, resulting in a deposition rate of $1-2 \times 10^{-2} \mathrm{~nm} / \mathrm{s}$ at a working pressure of $10^{-7} \mathrm{mbar}$. The samples were mounted at a substrate holder rotating at $1 \mathrm{~Hz}$ at a distance of $1 \mathrm{~m}$ from, and with the axis of rotation aligned with, the melt of target material. The low working pressure (leading to a $10^{3} \mathrm{~m}$ mean free path) and deposition geometry gives enhanced control and isotropy of the deposition flux compared to other deposition methods such as magnetron sputtering. The local angle of incidence of the deposition flux was constant during sample rotation and isotropic within $0.5^{\circ}$. Adjustments to the sample position and orientation were made to modify the local angles of incidence of the deposition flux: $\partial_{\text {depo }}=-2.65^{\circ}, 2.65^{\circ}$, and $3.95^{\circ}$ [Fig. $1(\mathrm{a})$ ].

\section{B. Characterization}

\section{Cross section transmission electron microscopy}

The cs-TEM specimens were prepared in sandwich shape, containing the sample under study and some supporting Si wafer material, making use of a two component resin. The cross section was taken perpendicular to the cleaving edge. A disk shaped sample was extracted and further processed by the method of dimpling-grinding/polishing, in order to minimize preparation induced artifact near the fragile edge region. For the final thinning of the sample by $\mathrm{Ar}^{+}$-etching, sequential processing/TEM-inspection cycle were performed to ensure that the edge region and the protecting resin remained intact. Given the phase of the multilayer structure TEM contrast is governed by mass differences. Therefore, images were recorded in bright-field conditions as to provide maximum contrast and the best possible visibility of any deviations from the periodicity of the $\mathrm{Mo} / \mathrm{Si}$ stack. Sample alignment was obtained along the (011) zone axis from the crystalline Si substrate. All measurements have been performed at an acceleration voltage of $300 \mathrm{kV}$ in a Philips CM300ST-FEG TEM, with focus on the step-edge region.
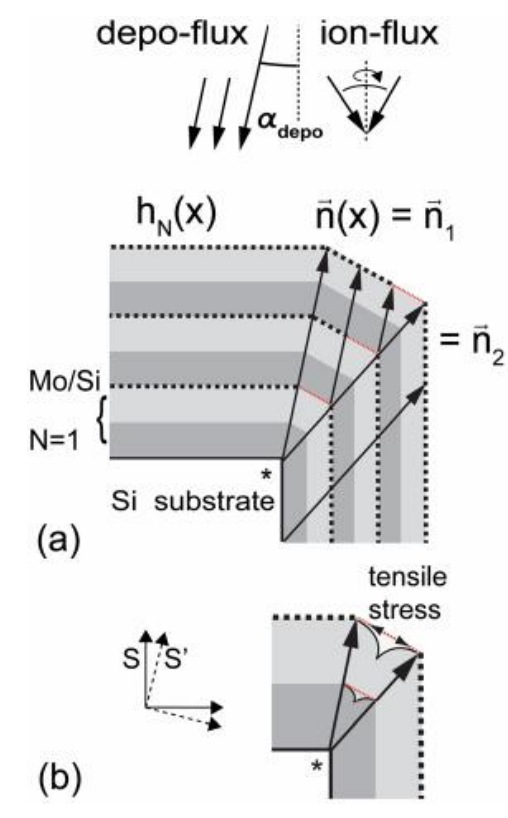

FIG. 1. (Color online) Schematic deposition geometry and recursive layer profiles for anisotropic layer growth into $n{ }^{\star} 1$ and $n 2$. $\left({ }^{\star}\right)$ Indicates stepedge under study. Thin (red) lines are interpolated data. 


\section{EUV reflectometry measurements}

Reflectance measurements were performed at the Center for X-ray Optics, making use of the Advanced Light Source synchrotron facility. At large distances from the step-edges, the reflectance spectrum around $13.5 \mathrm{~nm}$ and at $5^{\circ}$ from the local surface normal was measured. Reflectance measurements near the step-edge were performed employing the AIT; for a detailed description of the apparatus is referred to (see Ref. [9]). The step-edge regions of the samples were illuminated uniformly at wavelengths in the range 13-14 $\mathrm{nm}$ and $6^{\circ}$ from the sample plane normal. A zoneplate with a numerical aperture of 0.075 was used to project the reflected radiation from a diffraction limited spot onto a CCD camera at the back focal plane with a 907x magnification, providing an EUV intensity image of the sample with a spatial resolution better than $100 \mathrm{~nm}$. Per recorded image the measuring geometry was kept mechanically fixed, and the wavelength dependence of the focal length of the zoneplate was used to obtain through-focus image series. By adjusting the sample-zoneplate distance, two through-focus series were obtained with the multilayer surface in focus at different wavelengths. For straightforward comparison and highest spatial resolution, the images with the optical surface in-focus have been processed.

\section{Continuum model simulations}

The recursion relation [Eq. (1) to Eq. (2)] was numerically evaluated at an array of data points in a Cartesian coordinate system $S(x z)$ on a fixed uniform grid representing $h_{0}(x)$ as a (close to) $90^{\circ}$ step-edge (Fig. 1). By linear interpolation the data was projected at the initial grid where applicable, after each recursive step. This method allows modeling anisotropic layer growth by introducing spatially dependence in the layer growth front $\vec{n} \rightarrow \vec{n}(x)$ in Eq. (3), while maintaining the uniformity of the grid as required for the fast Fourier transformalgorithm used. To obtain ample sampling points at the step-edge side, for enhanced numerical stability of the algorithm, the Fourier transform was applied in a coordinate system $S$ ' which is rotated as compared to S. For practical reasons an angle of $\arctan (0.25)$ was chosen. It should be noticed that for a nonflat substrate the layer growth model does not imply a preferential coordinate system in which the Fourier transform should be evaluated; hence, S' can be chosen arbitrary as long as regarded as a model parameter.

For the described multilayer structure and deposition conditions two nonlinear contributions to Eq. (3) are considered relevant

$$
\begin{aligned}
\vec{\partial}_{n l}(x) & =\vec{\partial}_{\text {comp }}(x)+\vec{\partial}_{\text {sput }}(x) \\
\vec{\partial}_{\text {comp }}(x) & =C\{1-1 /(x) \cdot \vec{n}\} \text { and, } \\
\vec{\partial}_{\text {sput }}(x)=-t_{\text {sput }}(x) \cdot \vec{n}_{\text {sput }}-\bar{P}_{1} & \\
& \quad x \exp \left\{-p_{2} \bar{t}(x) \cdot \vec{n}_{\text {sput }} \bar{p}_{3}-1\right\} \vec{n}_{\text {sput }} .
\end{aligned}
$$

Equation (4) addresses compaction into the local surface normal direction at the multilayer interfaces. ${ }^{12}$ Equation (5) describes layer removal by physical sputtering resulting from the noble ion exposure in its simplest representation the process can be regarded as inverse growth with a layer front $\vec{n}_{\text {sput }}$. The above universal analytic expression was used to account for anisotropy in sputter yield as a function of local angle of incidence of the ions at the surface, with coefficients $p_{1}, p_{2}, p_{3}$. Corresponding to Ref. 10 the parameters in Eq. (5) were chosen $p_{1}=1.7, p_{2}=2.1$, and $p_{3}=2.4$. Moreover, sample rotation $(\omega \vec{z})$ introduced a $2 p$-periodic modulation in the local surface normal $\vec{\xi}(x) \rightarrow \vec{\xi}(x, \varphi)$, with $\omega t \equiv \varphi$, relative to the fixed $\mathrm{Kr}^{+}$ion sputtering geometry. The effective sputter yield therefore was calculated by averaging the scalar part of Eq. (5) over $0 \leq \varphi \leq 2 \pi$, yielding $\left\langle\partial_{\text {sput }}\right\rangle_{\varphi}(x)$. For regions in the shadow of the of 
the ion flux, $\vec{\xi}(x, \varphi) \cdot \vec{n}_{\text {sput }}<0$, the sputter yield was set to zero. The sample rotation results in a symmetric and more uniform effective sputter yield as a function of the surface tangent (Fig. 2).

\section{RESULTS AND DISCUSSION A. cS-TEM analysis}

Cross section TEM graphs of the multilayers at the step- edge region are shown in Fig. 3. At the resolution required for proper imaging the bilayer profiles show no indications of any influence of strain, dislocations, and vacancies are observed. In general, the influence of the discontinuous substrate on the multilayer structure predominantly manifests within a lateral distance 6 $100 \mathrm{~nm}\left(\mathrm{x}^{\star}\right)$ from the step-edge. A three-zone structure diagram is proposed for further discussion of the cs-TEM data.

The step-edge upper terrace expanded into the negative deposition direction defines zone (i). Here a continuous layer structure is observed, with slightly bended layer profiles near the zone boundary. A uniform layer growth front directed towards the deposition flux at the entire step-edge substrate implies lateral confinement of the semiparallel multilayer to zone (i). The observation of the multilayer extending beyond

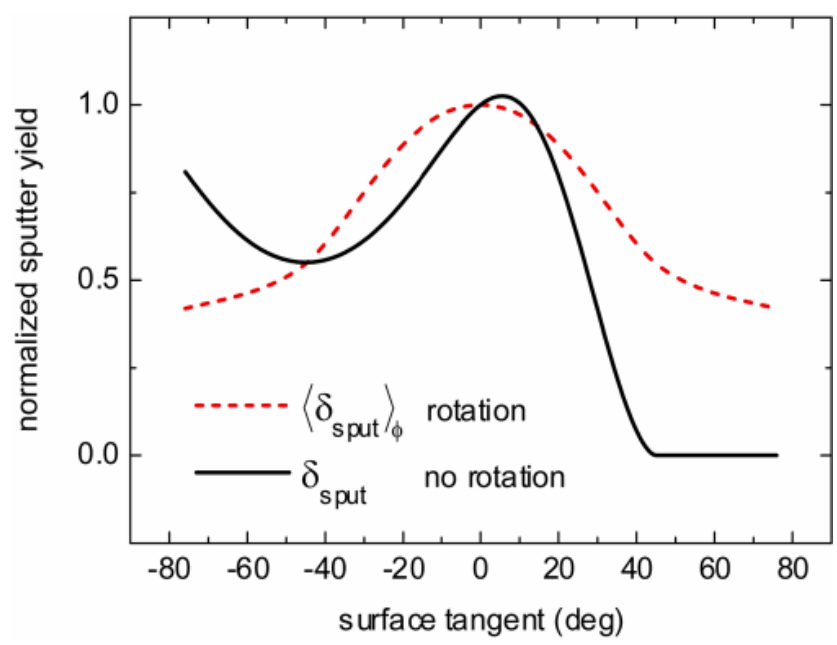

FIG. 2. (Color online) Normalized sputter yield [Eq. (3)] for $45^{\circ}$ angle of incidence ions relative to the $0^{\circ}$ surface tangent, with sample rotation (azimuthally averaged) and without. In the case of no rotation, ions propagate parallel to the surface for $\mathbf{P} 45^{\circ}$ surface tangent. 

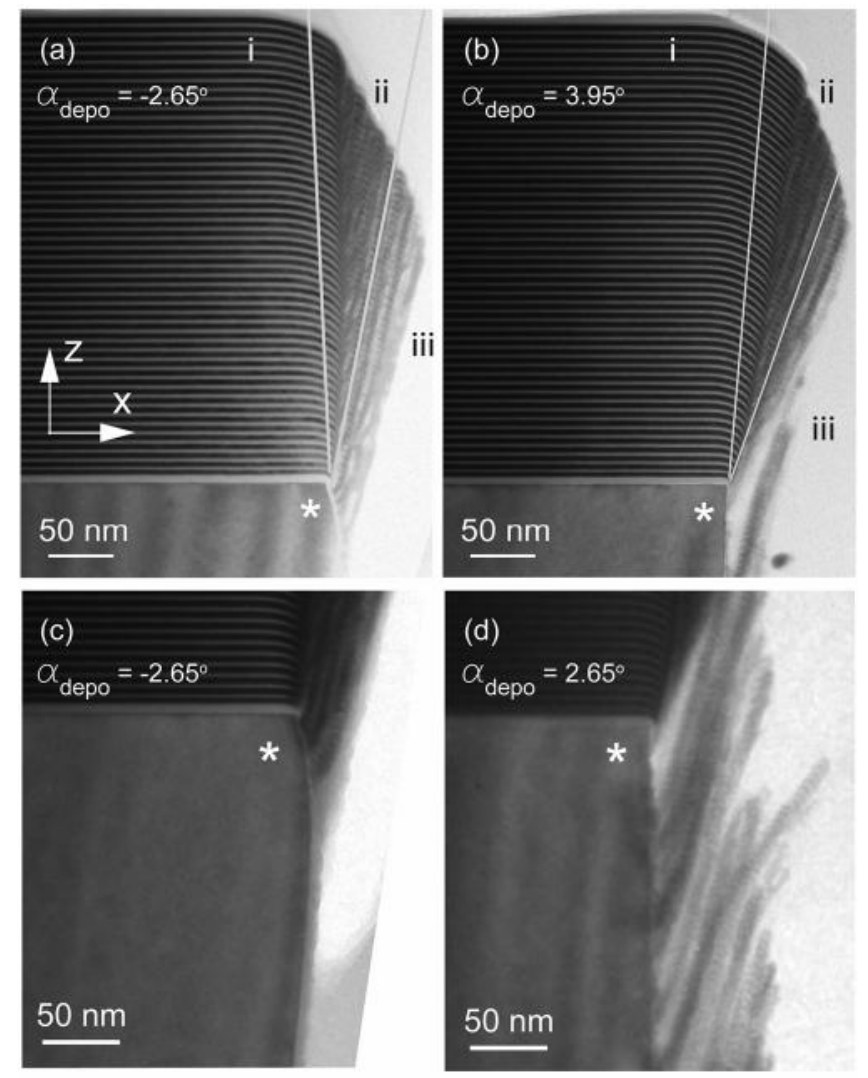

FIG. 3. Cross section TEM graphs of Mo/Si multilayers on cleaved Si wafers. Boundaries zone (i)-(ii)-(iii) indicated by the lines. (a) adepo $=-2.65^{\circ}$, (b) adepo $=3.95^{\circ}$, (c) - (d) details step-edge side unexposed and exposed to deposition flux, respectively.

the zone boundary (i) in positive $x$-direction, gives a first indication of anisotropic layer growth.

At zone (ii) a transition from the well-defined but increasingly bending layers, towards a disordered morphology in which besides the multilayered structure a columnar structure can be recognized, is apparent. Columnar layer growth is typical for the intensively studied regime of layer growth at glancing angle thin film PVD (Ref. 13). As is determined empirically from the csTEM graphs, the transition occurs at a critical local derivative of the upper layer profile function of approximately $\partial_{x} h(x)=-\tan \left(\alpha_{\text {depo }}+42^{\circ}\right) \equiv \partial_{x} h_{c o l}$. The orientation of the columns indicates a growth front inclined towards the local surface normal with an angle $\alpha_{\text {col }}$ measured from $\vec{z} ; \alpha_{c o l}=21^{\circ}$ for $\alpha_{\text {depo }}=2.65^{\circ}$ and $3.95^{\circ}$, and $\alpha_{c o l}=11^{\circ}$ for $\alpha_{\text {depo }}=-2.65^{\circ}$. The differences might not be attributed entirely to the deposition conditions, but also to the nonidentical step-edge shapes. The observations are in general agreement with the Lichter-Chen model on columnar layer growth, ${ }^{14}$ which is based on finite mobility of adparticles before condensation and self-shadowing of condensation nuclei.

The third zone covers the fully columnar layer structure, with corresponding layer growth front, as observed at the stepedge side. A clear view on this region is given by Fig. 3(c) and $3(\mathrm{~d})$. At $\partial_{\text {depo }}=-2.65^{\circ}$ the side was not exposed to the deposition flux while for $\partial_{\text {depo }}=2.65^{\circ}$ and $3.95^{\circ}$ the columnar structures are unambiguously observed. The columnar bilayers thickness is reduced as compared to the continuous multilayer structure: $d_{c o l}<d$. This may be caused by the disordered layer morphology and consequent large interface area, propagating (dense) compound formation.

The observations show strong analogies with reported behavior of other Bragg-reflector multilayer systems on grating topographies, such as La/B4C and Mo/B4C (Refs. 15 and 16). This justifies a broader applicability of the three zone structure diagram for a class of nanoscale multilayer systems other then Mo/Si. Suppression of columnar layer growth would be 
desirable for facilitating straightforward zone (i) substrate replication. An adjusted deposition geometry or substrate design could both be viable methods to achieve this, but the consequences to device design and functionality should be further examined.

\section{B. SIMULATION RESULTS}

The model parameters used in the simulations are given in Table I. The anisotropic layer growth directions are addressed, where the columnar layer growth occurs for surface profile derivatives $\leq \partial_{x} h_{c o l}$. A quantitative explanation of the columnar layer morphology is beyond the scope of the continuum model. The simulated data are therefore depicted up to the critical surface profile derivative $\partial_{x} h_{c o l}$. It is noted that hereby the extent to which the continuous layers overhang the edge is somewhat overestimated; in the experimental data the transition to the columnar layer is observed closer to the projected step-edge position (Fig. 4). The discrepancy may relate to the a priori assumption of continuous layer formation at zone (ii), as implied by the linear interpolation of data points between at the onset of columnar growth. Although not further investigated, continuous layer formation a the transition region is expected to be inhibited by high tensile stresses arising from the anisotropy in layer growth directions [Fig. 1(b)].

The gradually bending of each individual bilayer in the multilayer structure is accurately described for zone (i) and zone (ii), up to the point the continuity of the layers breaks. Fourier component relaxation in the surface profile functions is governed by viscous flow and free volume annihilation

TABLE I. Continuum model parameters as used in the simulation. Errors relate to uncertainties in experiment and data extraction from the cs-TEM graphs.

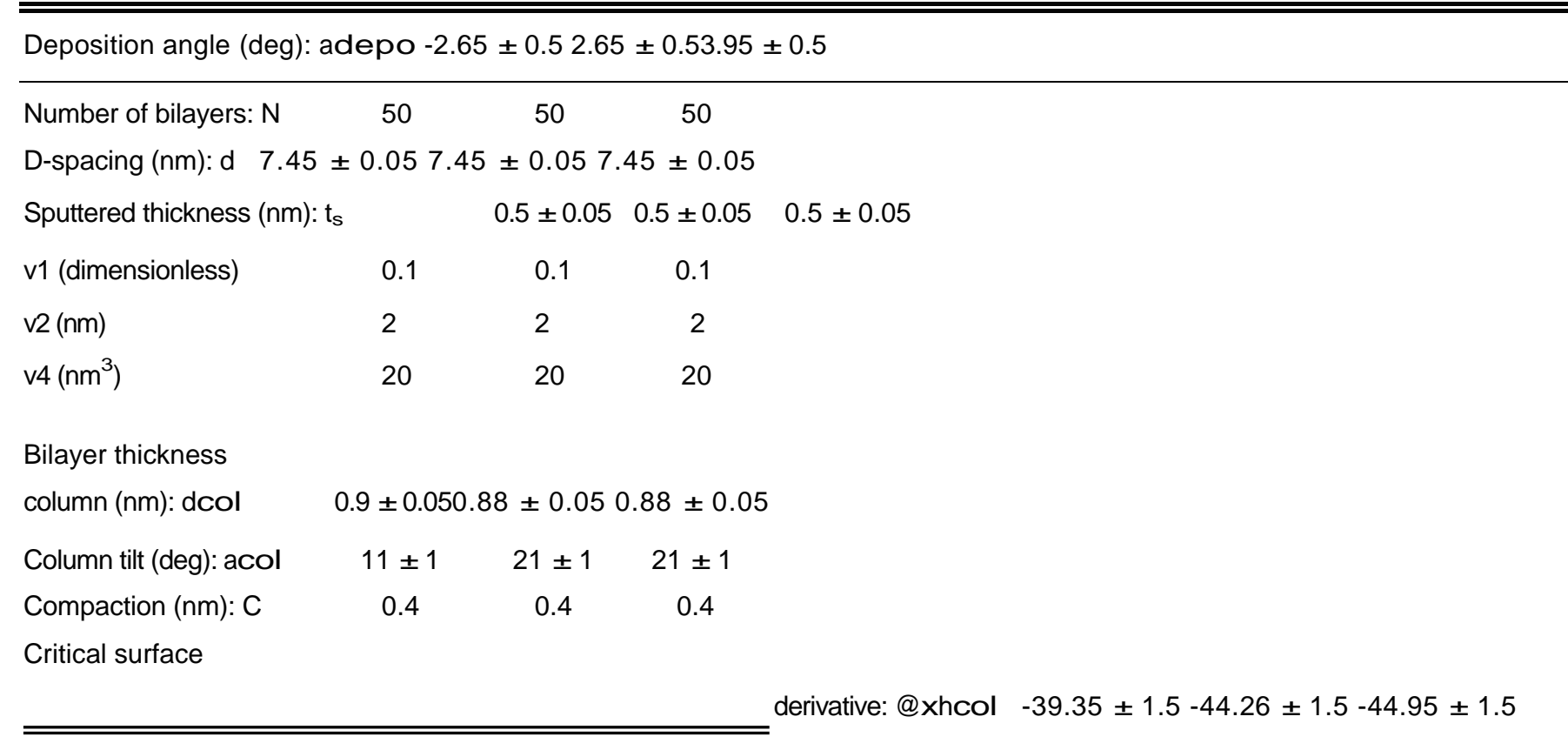



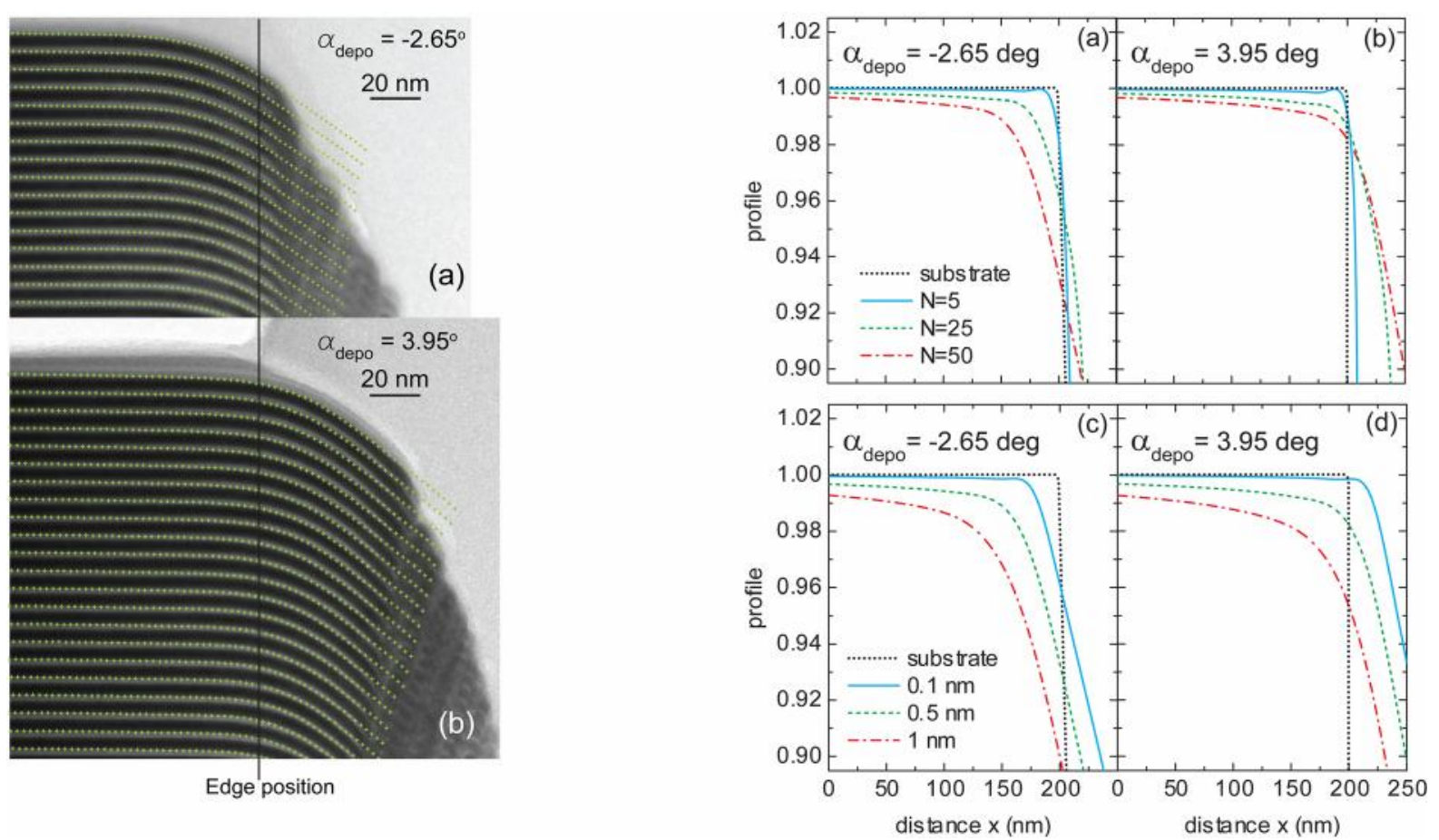

FIG. 4. (Color online) Comparison of simulations and data for model parameters in Table I. Cross section TEM data details 4(a) and 4(b) were obtained from the overview graphs in Fig. 3(a) and 3(b), respectively.

(a 2pf) (Ref. 17), sputtering and re-deposition [a (2pf)2] (Ref. 18), and surface confined viscous flow [a (2pf)4] (Refs. 19 and 20). The nonlinear terms given by Eq. (4) and Eq. (5) have a minor influence on the layer profile evolution at realistic parameter values.

Figure 5 represents the simulated layer profile functions at varying bilayers number. An increase in deposition angle mainly translates into a lateral shift of the layer profiles into the positive x-direction [Fig. 5(a) and 5(b)]. An enhanced flatness of the layer profiles at the step-edge region is observed for reduced ion-induced kinetics, of which the ion-sputtered layer thickness provides experimental control under presented deposition conditions [Fig. 5(c) and 5(d)]. Restriction of the ion-sputtering treatment will simultaneously reduce the mitigation of substrate replicated and layer growth intrinsic interface roughness. Depending on substrate roughness and multilayer composition this might compromise the Bragg-reflector quality.

\section{EUV reflectometry}

In the spectral EUV reflectance data obtained far from the step-edges, a maximum is observed at a wavelength of $13.57 \mathrm{~nm}$ [Fig. 6(a)]. Correspondingly, the d-spacing is found to be $6.98 \mathrm{~nm}$ as derived from Fresnel equation based calculations using the IMD computer code,$^{21}$ for a four-layer multilayer system with a layer of $0.6 \mathrm{~nm}$ and $1 \mathrm{~nm}$ of MoSi2 at the Si-on-Mo and Moon-Si interface, ${ }^{22}$ respectively, and 1.7 to $2 \mathrm{~nm}$ of native oxide at the Si terminated 50 bilayer stack.

Figure $6(\mathrm{~b})$ represents the in-focus AIT reflection profiles obtained near the step-edge, as a function of in-plane distance to the step-edge, for $\partial_{\text {depo }}=-2.65^{\circ}$ and $2.65^{\circ}$. The data have 
FIG. 5. (Color online) Normalized layer profiles based on parameters in Table I. (a)-(b) Various bilayer number (N). (c)-(d) Various ionsputtered thickness for $\mathrm{N}=50$.

been normalized on a first-order polynomial fit to the data at the region 0-500 $\mathrm{nm}$, to correct for small misalignment and beam aberrations. The reflection profiles are almost flat up to a point were the signal significantly increases, with a maximum increase of $\mathrm{Al}$, followed by a drop-off towards zero reflection. It should be noticed that the wavelength in the AIT experiments is shorter than the wavelength of maximal reflectance at a d-spacing of $6.98 \mathrm{~nm}$. Hence the increased reflected intensity at the sample edge indicates a closer matching of the Bragg-condition for constructive interference caused by a reduced d-spacing at the edge of the samples. IMD calculations show that the observed wavelength dependence in Al can be explained by assuming a decrease in $\mathrm{d}$ spacing of $\mathrm{Ad}=0.28 \%$ and $0.27 \%$ for $\operatorname{ad} \mathrm{d}_{\mathrm{pp}}=-2.65^{\circ}$ and $2.65^{\circ}$, respectively (Fig. 7 ). To match the calculations with the measured

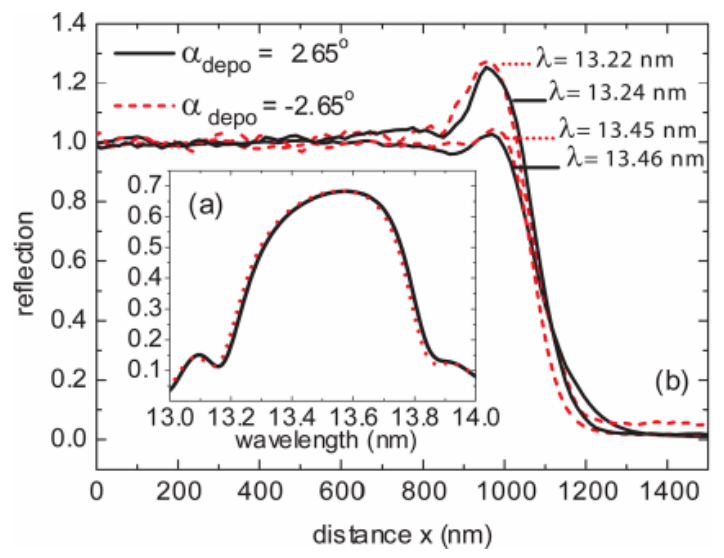

FIG. 6. (Color online) Reflectometry data, (a) wavelength scans far from the sample edge (b) AIT normalized reflection profiles at step-edge region 


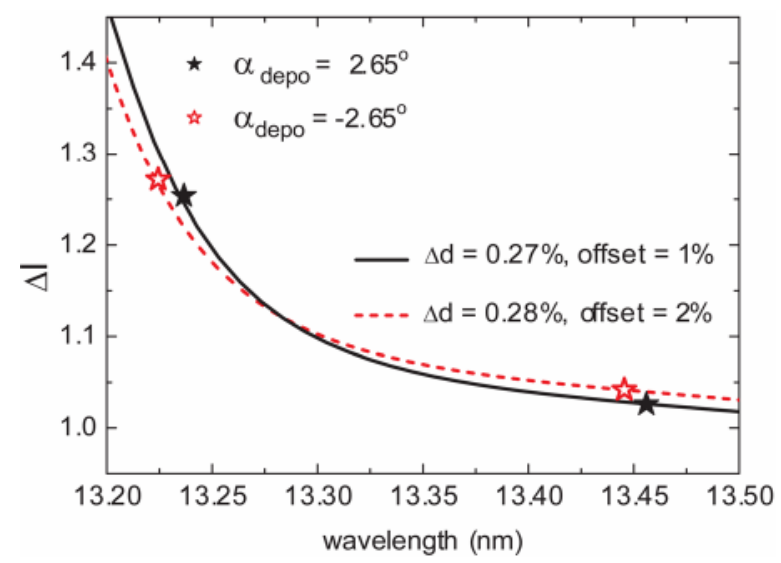

FIG. 7. (Color online) Increase in AIT reflection profiles near step-edges plotted against wavelength. IMD caluclations (lines) and data shown.

data, a small positive offset in the calculated DI curves has been applied. Edge-diffraction effects are considered of minor importance; since in-focus images were selected for data analysis a minimized range in interfering phases contributes to the recorded intensity. However, the offset values might be attributed to knife-edge diffraction.

The reflectometry results can be well understood in terms of the continuum model simulations, which show a decrease in dspacing of $0.3 \%$ for an almost unchanged surface normal at $125 \mathrm{~nm}$ from the step-edge (Fig. 5), without strong dependence on adepo. This position is directly related to the maximum reflectance in the AIT data. An even stronger bilayers thickness decrease, as observed in the cs-TEM images near the zone boundary (i)-(ii), implies an oblique surface normal and therefore misalignment in the AIT measurements, yielding a close to zero reflection. Considering the width of the peak in the AIT signal near the stepedge, the region for which the d-spacing is significantly reduced is estimated to be restricted to $300 \mathrm{~nm}$ from the cleaved wafer edge position.

\section{SUMMARYAND CONCLUSIONS}

$\mathrm{Mo} / \mathrm{Si}$ multilayer structures have been deposited on stepped substrate topographies for isotropic deposition fluxes at various, close to normal, angles of incidence. The height profiles of the layers were studied by cs-TEM. The data are indicating continuous layer growth into the deposition direction at the upper step-edge terrace [zone(i)], a transition regime from continuous to columnar layer growth when approaching the step-edge [zone(ii)], and a fully columnar regime at the stepedge side [zone(iii)]. The occurrence of the columnar layer growth is explained by the deposition conditions and geometry.

By applying a simple continuum model the layer profiles functions can be quantitatively described for the continuous layer regimes [zone(i) and (ii) up to the transition point]. It accounts for the deposition geometry, ion induced kinetics, compound formation, and most notably the anisotropic layer growth directions as associated with the transition from continuous to columnar layer growth. The observed gradual bending of the layer profiles at zone(i) and zone(ii) strongly depends on the ion enhanced deposition conditions.

Extreme ultraviolet reflection measurements using the Actinic Inspection Tool (AIT) have been used to measure the optical performance of the multilayers close to the step- edge and show a slightly affected reflectance at the step-edge region. This effect is related to a decrease in d-spacing of slightly less than $0.3 \%$. The latter occurs at a distance of $125 \mathrm{~nm}$ from the step-edge, whereas the measurable optical perturbation of the planar multilayer is restricted to a region within $300 \mathrm{~nm}$ from the stepedge, as is quantitatively derived by comparison of the AIT data with the continuum model simulation.

\section{ACKNOWLEDGMENTS}

We acknowledge financial support from the AgentschapNL through the EXEPT and ACHieVE programs coordinated by 
ASML and the Foundation for Fundamental Research on Matter (Stichting voor Fundamenteel Onderzoek der Materie, FOM) and Carl Zeiss SMT GmbH through the Industrial Partnership Program XMO. Furthermore, the authors wish to thank E.G. Keim of the MESAP Institute for Nanotechnology for the TEM analysis, E.M. Gullikson at the Center for X-ray Optics for EUV reflectometry measurements, and $\mathrm{B}$. LaFontaine at Cymer for making experimental time available on the AIT.

1J. A. Liddle, F. Salmassi, P. P. Naulleau, and E. M. Gullikson, J. Vac. Sci. Technol. B 21, 2980(2003).

${ }^{2}$ D. L. Voronov, M. Ahn, E. H. Anderson, R. Cambie, C.-H. Chang, E. M. Gullikson, R. K. Heilmann, F. Salmassi, M. L. Schattenburg, T. Warwick, V. V. Yashchuk, L. Zipp, and H. A. Padmore, Opt. Lett. 35, 15 (2010).

${ }^{3}$ I. V. Kozhevnikov, R. van der Meer, H. M. J. Bastiaens, K.-J. Boller, and F. Bijkerk, Opt. Express 18, 15 (2010).

${ }^{4}$ A. M. Nugrowati, A. S. van de Nes, S. F. Pereira, and J. J. M. Braat, Microelectron. Eng. 83, (2006).

${ }^{5}$ D. L. Voronov, E. H. Anderson, R. Cambie, S. Dhuey, E. M. Gullikson,

F. Salmassi, T. Warwick, V. V. Yashchuk, and H. A. Padmore, Nucl. Instrum. Methods A, 649, 156 (2010).

${ }^{6}$ W. Hu, M. Zhang, R. J. Wilson, A. Leen Koh, J.-S. Wi, M. Tang, R. Sinclair, and S. X. Wang, Nanotechnology 22,185302 (2011).

${ }^{7}$ E. M. Gullikson and D. G. Stearns, Phys. Rev. B 59, 20(1999). ${ }^{8}$ D. G. Stearns, Appl. Phys. Lett. 62 , 1745 (1993).

${ }^{9}$ K. A. Goldberg, I. Mochi, P. Naulleau, T. Liang, P.-Y. Yan, and S. Huh, J. Vac. Sci. Technol. B 27, 2916(2009). 10D . G. Stearns, P. B. Mirkarimi, and E. Spiller, Thin Solid Films 446,37(2004).

${ }^{11}$ R. W. E. van de Kruijs, E. Zoethout, A. E. Yakshin, I. Nedelcu, E. Louis,

H. Enkisch, G. Sipos, S. Müllender, and F. Bijkerk, Thin Solid Films 515, 430(2006).

${ }^{12}$ R. S. Rosen, D. G. Stearns, M. A. Viliardos, M. E. Kassner, S. p. Vernon, andY. Cheng, Appl. Opt. 32,34 (1993).

${ }^{13}$ M. M. Hawkey and M. J. Brett, J. Vac. Sci. Technol. A 25, 5 (2007). ${ }^{14}$ S. Lichter and J. Chen, Phys. Rev. Lett. 56,13 (1986).

${ }^{15}$ D. Häussler, E. Spiecker, S. Yang, W. Ja"ger, M. Stormer, R. Bormann, G. Zwicker, Status Solidi A 202, (2005).

${ }^{16}$ D. Ha"ussler, E. Spiecker, W. Ja"ger, M. Stormer, R. Bormann, C. Michaelsen, J. Wiesmann, G. Zwicker, R. Benbalagh, J.-M. Andre', and P. Jonnard, Micro- electron. Eng. 84, (2007).

${ }^{17}$ A. J. R. van den Boogaard, E. Louis, E. Zoethout, S. Müllender, and F. Bijkerk, J. Vac. Sci. Technol. A 28, 4 (2010).

${ }^{18}$ M. Moseler, P. Gumbsch, C. Casiraghi, A. C. Ferrari, and J. Robertson, Science 309, 2 (2005).

${ }^{19}$ S. E Orchard, Appl. Sci. Res., Sect. A 11, 451 (1962).

${ }^{20}$ C. C. Umbach, R. L. Headrick, and K.-C. Chang, Phys. Rev. Lett. 87, 24 (2001).

${ }^{21}$ D. L. Windt, Comput. Phys. 12, 4 (1998).

${ }^{22}$ A. E. Yakshin, E. Louis, P. C. Gorts, E. L. G. Maas, and F. Bijkerk, Physica B 283, 143 (2000). 


\section{DISCLAIMER}

This document was prepared as an account of work sponsored by the United States Government. While this document is believed to contain correct information, neither the United States Government nor any agency thereof, nor The Regents of the University of California, nor any of their employees, makes any warranty, express or implied, or assumes any legal responsibility for the accuracy, completeness, or usefulness of any information, apparatus, product, or process disclosed, or represents that its use would not infringe privately owned rights. Reference herein to any specific commercial product, process, or service by its trade name, trademark, manufacturer, or otherwise, does not necessarily constitute or imply its endorsement, recommendation, or favoring by the United States Government or any agency thereof, or The Regents of the University of California. The views and opinions of authors expressed herein do not necessarily state or reflect those of the United States Government or any agency thereof or The Regents of the University of California.

This work was supported by the Director, Office of Science, of the U.S. Department of Energy under Contract No. DE-AC02-05CH11231. 